\title{
Hunting for Foxes with Sheaves
}

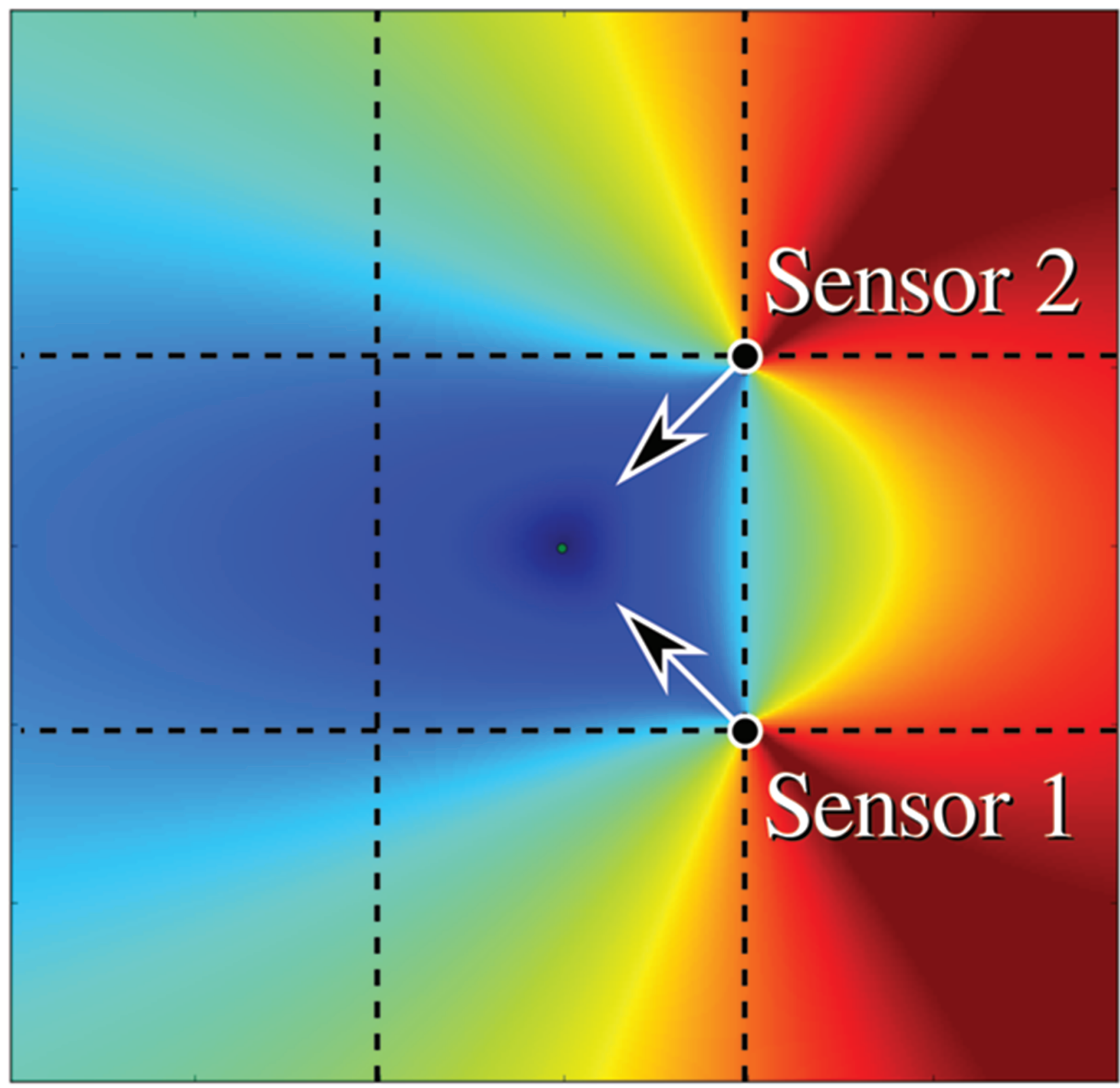

Michael Robinson

Michael Robinson is an Associate Professor in the Department of Mathematics and Statistics at American University. His email address is michae1r Qamerican.edu.

All figures can be reproduced using The Jupyter Notebook at: https: //gi thub .com/kb1dds/foxsheaf.

Communicated by Notices Associate Editor Emilie Purvine.

\section{Introduction}

To a radio amateur (or "ham"), fox hunting has nothing to do with animals. It is a sport in which individuals race

For permission to reprint this article, please contact:

reprint-permission@ams . org.

DOI: https://doi.org/10.1090/noti1867 
each other to locate a hidden radio transmitter on a known frequency. Since hams are encouraged to design and build their own equipment, the typical fox hunt involves a variety of different receivers and antennas with different capabilities. Some of these can display the received signal strength from the hidden transmitter (loosely measuring distance to the transmitter), while others estimate the compass bearing. Both of these estimates vary in accuracy and in precision depending on terrain, environmental conditions, equipment quality, and the skill of the operator.

Fox hunting also serves the purpose of preparing radio amateurs for emergency or disaster operations. Because disaster operations require the concerted efforts of multiple radio operators, it seems fitting to explore how the sport changes if fox hunting becomes cooperative. When participants combine their estimates of distance and bearing, how much faster can they find the transmitter? Part of the challenge of fox hunting is that measurements are taken infrequently, only once every few minutes. To win the hunt, every minute must count! In the most demanding scenario, each sensor only gets to take one measurement of the fox transmitter.

Locating the fox transmitter from a collection of different sensors is a model-based data fusion problem: combining disparate local observations into a global inference. Without a model that describes how signals from the transmitter arrive at each receiver, the signal reports are not helpful for locating the transmitter. Even with such a model, the effects of terrain, the transmitter's antenna system, and the environment can cause substantial differences between the modeled signal and an actual received signal. Therefore, it is important that we remain even-handed about assumptions of the quality of the estimates and the quality of the model.

Though there are many techniques for solving data fusion problems, they broadly fall into two categories: (1) problem-specific deterministic methods, and (2) general statistical methods. Well-crafted problem-specific deterministic methods are very effective. Because of the physics of radio propagation, it is not too difficult to construct a deterministic method specifically for locating a fox transmitter. However, problem-specific methods often carry hidden assumptions that make it hard to transfer useful techniques to another problem. Worse, the bookkeeping associated with all combinations of sensors grows exponentially as more sensors are deployed. Regardless of their attraction, problem-specific methods for solving data fusion problems are costly and difficult to manage. Statistical methods automate the bookkeeping and tailoring needed for a problem-specific method, but they usually require many observations to produce accurate results. Since we are only using one measurement from each sensor, statistical methods are not the best option.
We are left with the need for a general deterministic method for finding the fox from a small number of measurements. This article explains how to meet this need using sheaves, mathematical objects that describe local consistency within data. We can perform data fusion for any sheaf, though the fox hunting problem will guide our selection of the specific sheaf we need and will be the context for its interpretation. The resulting fox hunting sheaf is modular; different sensors or models of their performance can be substituted easily without changing how their data are analyzed.

This article explains how to model a collection of sensors in the section called "Formalizing the Sensors in the Fox Hunt" so that they can be combined into a sheaf model in "Formalizing the Interactions between Sensors." Once the sheaf is constructed, we show how to locate the fox transmitter in "Consistency Radius: Where is the Fox?" and determine if there are actually multiple fox transmitters in "Local Consistency Radius: Finding Multiple Foxes." Finally, since applied sheaf theory is still in its infancy, "Frontiers"points the reader to some interesting directions for future study.

\section{Formalizing the Sensors in the Fox Hunt}

Each receiver (or sensor) $A$ used in the fox hunt produces a signal report concerning its observation of the fox transmitter. Signal reports may be of different types, depending on the sensor. For instance, the strength of the received signal is typically reported as a single real number. In contrast, a compass bearing is reported as an angle, properly an element of the metric space $S^{1}$-the unit circle. To handle both of these situations (and more), let us suppose that a signal report is an element of a pseudometric space $D_{A}$, depending on the sensor $A$. The sensor $A$ produces reports through a continuous measurement function

$$
M_{A}: \mathbb{R}^{2} \times C_{F} \times \mathbb{R}^{2} \times C_{A} \rightarrow D_{A},
$$

depending on fox transmitter location (in the plane), the fox transmitter equipment settings $C_{F}$ (such as transmitter power and antenna orientation), the receiver location in the plane, and the receiver equipment settings $C_{A}$ (such as antenna orientation).

Our sensor data will be drawn from a parameterized distribution $S_{A}$, in which the noise level $\sigma$ is taken as a parameter. To ensure consistency between the deterministic model and the stochastic one, our stochastic models satisfy

$$
S_{A}\left(x, y, c_{f}, x^{\prime}, y^{\prime}, c_{A} ; \sigma\right) \rightarrow \delta_{M_{A}\left(x, y, c_{f}, x^{\prime}, y^{\prime}, c_{A}\right)} \text { as } \sigma \rightarrow 0,
$$

in which $\delta_{\alpha}$ is the unit impulse at $\alpha$.

There are typically two kinds of sensors that are used in radio fox hunting: calibrated signal strength meters and directional antennas. Given knowledge of the fox transmitter's power output, a calibrated signal strength meter 


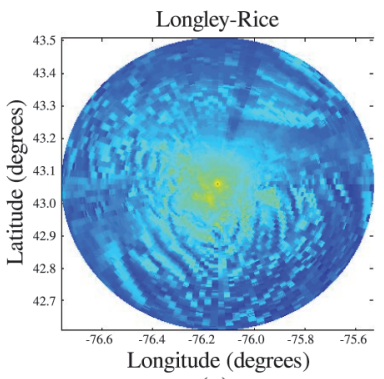

(a)

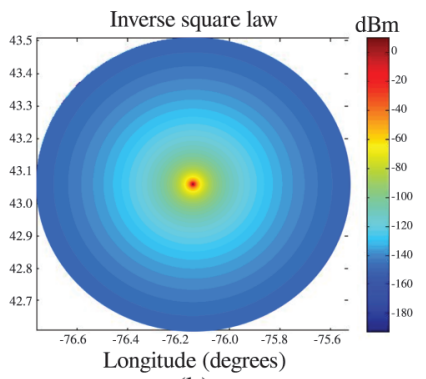

(b)
Figure 1. Simulated received power for a transmitter located at the top of a mountain using (a) the Longley-Rice model [8] and (b) the simplified model in (1). Colors are on a logarithmic scale, in decibels referenced to the power of 1 milliwatt $(\mathrm{dBm})$.

can help estimate the distance from the receiver to the fox. A directional antenna tells the operator the direction from which the fox's signal appears to be the strongest.

Calibrated signal strength (RSSI) sensors. A received signal strength indication (RSSI) sensor measures the amount of power absorbed by its antenna from the fox transmitter. To model this accurately requires careful specification of the terrain and any obstacles between the transmitter and receiver. The Longley-Rice model is popular among engineers because it incorporates the effect of terrain and atmospheric losses on the received power, and its predictions are realistic [8]. Figure 1(a) shows the received signal power predicted by the Longley-Rice model for a fox transmitter placed on a mountain.

While the realism of the Longley-Rice model is a benefit, its precise specification is quite complicated. None of this complexity is necessary to demonstrate our approach, because all our analyses are modular. A different measurement function can be substituted later if desired without changing the analysis techniques. Therefore, we will simply model the power transferred from transmitter to receiver by an inverse-square law (Figure 1(b)). This corresponds to a measurement function in which there is only fox transmitter power $p \in C_{F}=[0, \infty)$ and no receiver configuration,

$$
M_{R S S I}\left(x, y, p, x^{\prime}, y^{\prime}\right)=\frac{p}{4 \pi\left(\left(x-x^{\prime}\right)^{2}+\left(y-y^{\prime}\right)^{2}\right)},
$$

where $(x, y)$ is the transmitter location and $\left(x^{\prime}, y^{\prime}\right)$ is the receiver location.

From a statistical perspective, after the inverse square law, the next largest effect on the received signal is caused by self-interference as the signal travels along nearby paths with slightly different lengths. This effect is called Rician fading and is governed by the Rice distribution for signal amplitude (usually voltage) $V \in[0, \infty)$

$$
\operatorname{Rice}(V ; v, \sigma)=\frac{V}{\sigma^{2}} \exp \left(\frac{-\left(V^{2}+v^{2}\right)}{2 \sigma^{2}}\right) I_{0}\left(\frac{\nu V}{\sigma^{2}}\right)
$$

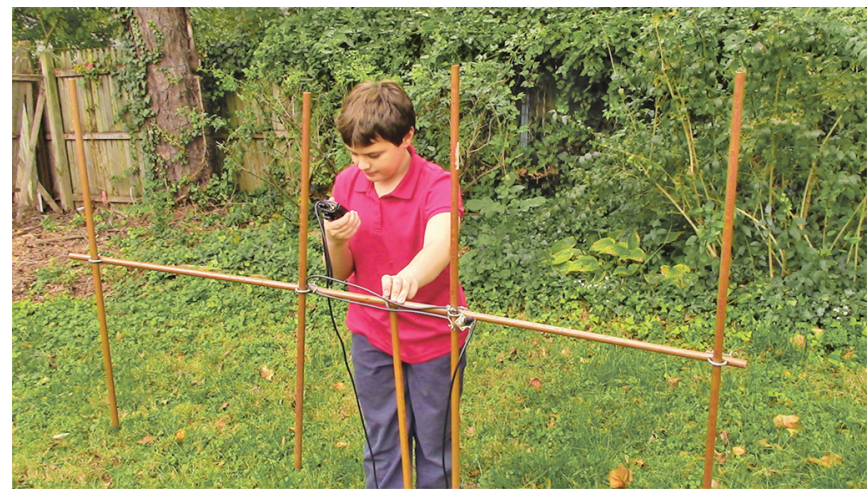

Figure 2. The author's son holding a direction-sensitive antenna (a Yagi-Uda array) built by the author for $144 \mathrm{MHz}$.

where $I_{0}$ is the modified Bessel function of the first kind. The Rice distribution models the distance between the origin and a point in the plane drawn from a bivariate Gaussian with mean $v$ and standard deviation $\sigma$. Assuming the received signal is a combination of the inverse square law and Rician fading, the RSSI signal reports $P$ will follow the distribution

$$
\begin{aligned}
& S_{R S S I}\left(P ; x, y, p, x^{\prime}, y^{\prime}\right) \\
& =\frac{1}{2 R}\left(\operatorname{Rice}\left(P ; \sqrt{2 M_{R S S I}\left(x, y, p, x^{\prime}, y^{\prime}\right) R}, \sigma\right)\right)^{2},
\end{aligned}
$$

in which antenna characteristic impedance $R$ (typically $50 \Omega$ for amateur equipment) and root mean square noise voltage $\sigma$ are assumed to be known in advance and constant. Bearing sensors. A bearing sensor measures the angle between true north (or some other convenient, global direction) and the apparent direction of arrival of signals from the fox transmitter. Most amateur bearing sensors consist of an antenna (like the one shown in Figure 2) that is preferentially sensitive to signals arriving from a specific direction. The operator rotates the antenna until the signal strength is greatest, and then records its direction. For strong signals, the operator can also block incoming signals by holding a hand held radio against the chest. When the operator slowly turns around until the signal strength is minimized, the transmitter is then directly behind the operator!

A useful antenna for fox hunting produces a response like one of the two shown in Figure 3 (p.664). It is a continuous, unimodal function on the circle $S^{1} \rightarrow \mathbb{R}$, since such a function has a single maximum (or minimum) in the direction of the fox transmitter (or in the exact opposite direction). A key performance criterion for an antenna is its beamwidth, the length of the interval for which the received signal exceeds half of the maximum value. In the presence of noise, an antenna with a small beamwidth will give more accurate bearing readings. 
Table 1. Independent sensors for the fox hunting examples

\begin{tabular}{|c|c|c|c|c|}
\hline Sensor ID & Location & Type & Measurement & Noise model \\
\hline \hline 1 & \multirow{2}{*}{$(1.0,0.0)$} & Position & $\mathbb{R}^{2}$ & (None) \\
\cline { 3 - 5 } & & Bearing & $S^{1}$ & von Mises \\
\hline 2 & \multirow{2}{*}{$(1.0,1.0)$} & Position & $\mathbb{R}^{2}$ & (None) \\
\cline { 3 - 5 } & & Bearing & $S^{1}$ & von Mises \\
\hline \multirow{2}{*}{3} & $(0.0,0.0)$ & Position & $\mathbb{R}^{2}$ & (None) \\
\cline { 3 - 5 } & & Signal strength & $\mathbb{R}$ & Rician \\
\hline \multirow{2}{*}{4} & \multirow{2}{*}{$(0.0,0.5)$} & Position & $\mathbb{R}^{2}$ & (None) \\
\cline { 3 - 5 } & & Signal strength & $\mathbb{R}$ & Rician \\
\hline
\end{tabular}

We will model the observations recorded by a bearing sensor stochastically by way of the von Mises distribution, the probability distribution that is the closest analogue of the Gaussian distribution for the circle [9]. The von Mises distribution is given by

$$
\operatorname{VonMises}(\theta ; \mu, \kappa)=\frac{e^{\kappa \cos (\theta-\mu)}}{2 \pi I_{0}(\kappa)}, \text { for } \theta \in S^{1}
$$

where again $I_{0}$ is the modified Bessel function of the first kind. By analogy with the Gaussian distribution, $\mu$ in the von Mises distribution plays the role of the mean, while $1 / \kappa$ is analogous to the variance. In order to model the measured bearing $\theta$ for a fox transmitter located at the true bearing $\mu$ from an antenna with beamwidth $B$, we set $\kappa=$ $4 / B^{2}$.

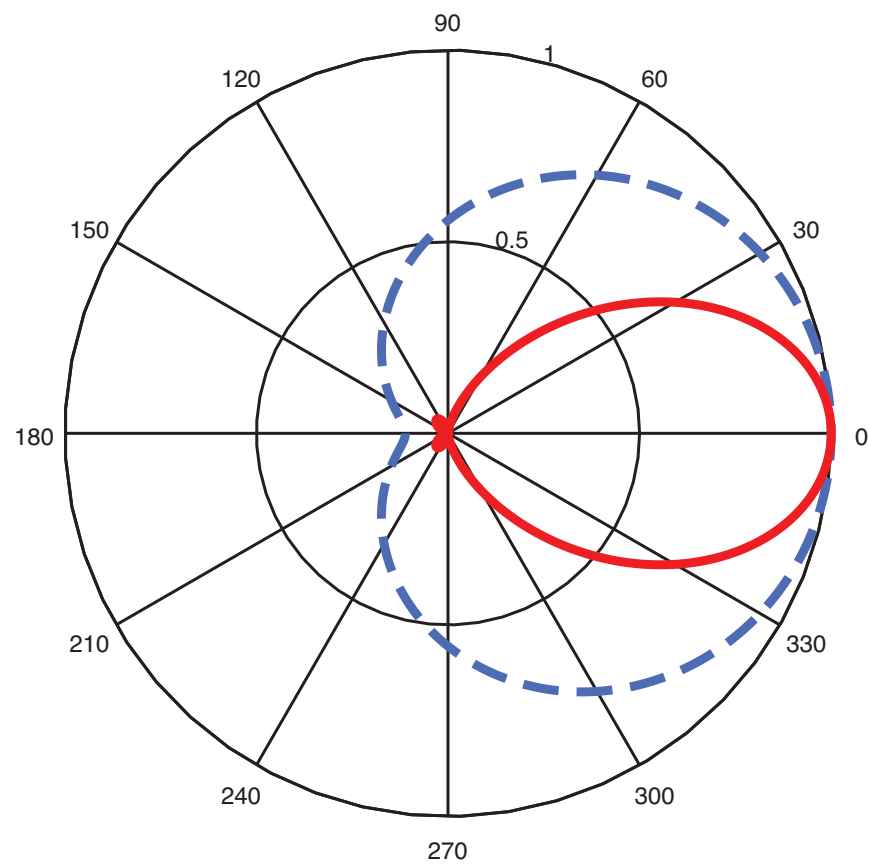

Figure 3. Simulated received signal strength as a function of angle for typical antennas used in fox hunting. The solid red curve is typical for a directional antenna like the one shown in Figure 2, while the dashed blue curve is typical for a handheld receiver held against the body.
Given a fox transmitter located at $(x, y)$ and sensor located at $\left(x^{\prime}, y^{\prime}\right)$, the true bearing (in degrees) can be obtained by the measurement function

$$
\begin{aligned}
& M_{\text {bearing }}\left(x, y, x^{\prime}, y^{\prime}\right) \\
& = \begin{cases}\frac{180}{\pi} \tan ^{-1} \frac{x-x^{\prime}}{y-y^{\prime}} & \text { if } y-y^{\prime}>0 \\
\frac{180}{\pi} \tan ^{-1} \frac{x-x^{\prime}}{y-y^{\prime}}+180 & \text { if } y-y^{\prime}<0 \text { and } x-x^{\prime} \geq 0 \\
\frac{180}{\pi} \tan ^{-1} \frac{x-x^{\prime}}{y-y^{\prime}}-180 & \text { if } y-y^{\prime}<0 \text { and } x-x^{\prime}<0 \\
90 & \text { if } y-y^{\prime}=0 \text { and } x-x^{\prime}>0 \\
-90 & \text { if } y-y^{\prime}=0 \text { and } x-x^{\prime}<0\end{cases}
\end{aligned}
$$

where true north is oriented along the positive $y$-axis and angles are measured clockwise. This simple model ignores some important effects, such as the fact that the apparent bearing can be distorted by reflections, but it is a good model for relatively flat terrain. Then the appropriate stochastic model is

$$
\begin{aligned}
& S_{\text {bearing }}\left(\theta ; x, y, x^{\prime}, y^{\prime}\right) \\
& \quad=\operatorname{VonMises}\left(\theta ; M_{\text {bearing }}\left(x, y, x^{\prime}, y^{\prime}\right), 4 / B^{2}\right)
\end{aligned}
$$

for an antenna with a known, constant beamwidth $B$.

Coordinating multiple sensors. Consider a team of four radio operators each equipped with a position sensor (a GPS receiver) that records their own location and a sensor that measures either the received signal strength from or the bearing to the fox transmitter according to Table 1 . Since GPS errors are small compared to those reported from amateur radio equipment, we will assume that GPS positions are known exactly. In all of the examples, we use the unit-less positions shown in the table rather than the latitude and longitude that would be reported by a GPS receiver.

Using this configuration of sensors, we will study five Cases (Table 2) that address two questions: (1) "Where is the fox?" and (2) "Are there multiple foxes?" We will start our analysis of the first question using Cases 1 and 2, before we demonstrate it with different realizations of stochastic noise in Case 3. Cases 4 and 5 will be used to address the second question. 
Table 2. Fox hunting cases

\begin{tabular}{|c||c|c|c|c|}
\hline Case & Sensors & $\begin{array}{c}\text { Number } \\
\text { of foxes }\end{array}$ & $\begin{array}{c}\text { Fox location } \\
\text { known }\end{array}$ & $\begin{array}{c}\text { Noise } \\
\text { present }\end{array}$ \\
\hline \hline 1 & All & 1 & Yes & No \\
\hline 2 & $\begin{array}{c}\text { Sensors 1 \& 2 } \\
\text { (bearings only) }\end{array}$ & 1 & Yes & No \\
\hline 3 & All & 1 & No & Yes \\
\hline 4 & $\begin{array}{c}\text { Sensors 1 \& 2 } \\
\text { (bearings only) }\end{array}$ & 2 & No & No \\
\hline 5 & All & 2 & No & No \\
\hline
\end{tabular}

\section{Formalizing the Interactions between Sensors}

Neither of the measurement functions $\left(M_{R S S I}\right.$ in (1) and $M_{\text {bearing }}$ in (3)) alone are sufficient to determine the location of the fox transmitter. It is only when multiple measurements from different locations or when different sensors' measurements are taken together that the location of the fox may be determined. This joint interaction between sensors can be encoded as a sheaf of pseudometric spaces on a partial order, by taking account of deterministic, functional dependencies between sensors' observations. Observations are of two types: (1) true observations: those actually made by sensors, and (2) virtual observations: those that could have been observed, but were not actually reported. The location and the transmitter power of the fox transmitter are in the latter class: the operator who hides the fox transmitter is legally required to observe its location and output power, but keeps these observations secret during the fox hunt!

It is reasonable to record position and a radio measurement simultaneously, so the typical measurement reported by Sensor 1 (a bearing sensor) will be of type $\mathbb{R}^{2} \times S^{1}$.

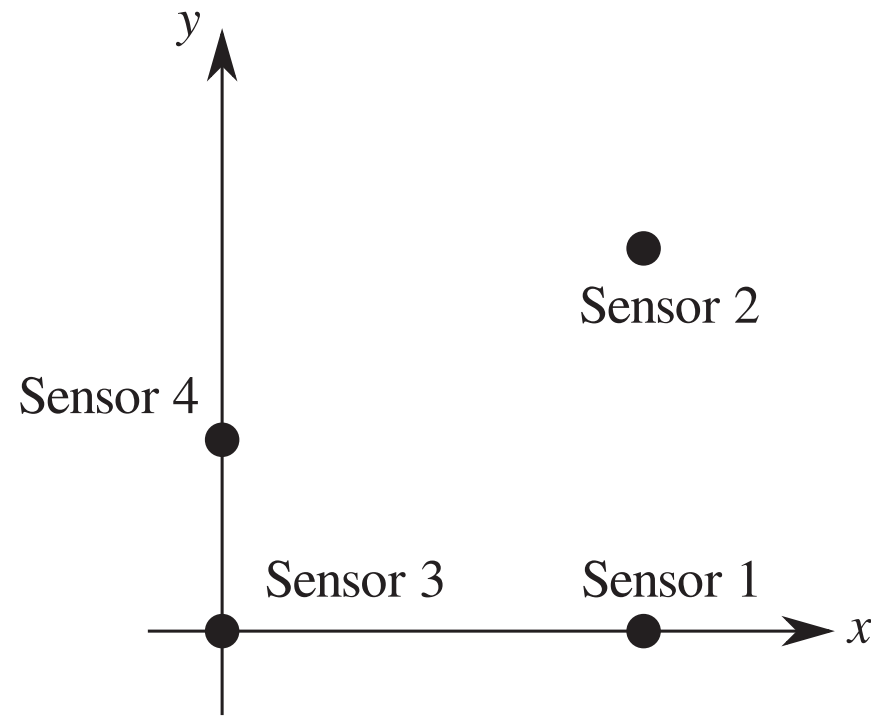

Figure 4. Spatial layout of the fox hunting examples
Table 3. Observations used in the fox hunting examples

\begin{tabular}{|c|c|l|}
\hline Observation & Virtual & Description \\
\hline \hline$F^{\prime}$ & Yes & Fox position, transmit power \\
$F$ & Yes & Fox position \\
$B_{1}^{\prime}$ & Yes & Fox position, Sensor 1 position \\
$B_{1}$ & No & Sensor 1 position and bearing \\
$B_{2}^{\prime}$ & Yes & Fox position, Sensor 2 position \\
$B_{2}$ & No & Sensor 2 position and bearing \\
$R_{3}^{\prime}$ & Yes & Fox position, Sensor 3 position \\
$R_{3}$ & No & Sensor 3 position and RSSI \\
$R_{4}^{\prime}$ & Yes & Fox position, Sensor 4 position \\
$R_{4}$ & No & Sensor 4 position and RSSI \\
\hline
\end{tabular}

This is a good strategy because there may be a statistical dependence between the position errors and the signal measurements. There are three observations that can be made: (1) the joint fox transmitter location and sensor location, (2) the fox transmitter's location, and (3) the joint bearing sensor's location and reported bearing. The first of these completely and functionally determines the other two, a situation that can be expressed as a partial order on the observations, which is shown in Figure 5(a) (p. 666). The joint fox transmitter location and sensor location (the minimal element in the partial order) is a virtual observation because we cannot make that joint observation without observing both simultaneously. The arrows in the diagram point from smaller to larger elements in the partial order, and express functional dependence. The spaces of observations and the actual functions themselves are shown in Figure 5(b), where $\operatorname{pr}_{k}\left(x_{1}, x_{2}, \ldots\right)=x_{k}$ is the projection from a product onto its $k$-th factor. In much the same way, the relationship for an RSSI sensor is between a virtual observation (the fox transmitter location, power level, and sensor location, jointly) and two true observations is shown in Figure 6 (p. 666). Measurement functions play the role of transforming virtual observations into true observations; if a different measurement function is desired, it can be easily substituted without disrupting the partial order.

Combining all four sensors under the hypothesis that all of the fox transmitters are actually the same yields a somewhat larger collection of observations, shown in Table 3 along with their interpretations. They are named so that $B$ is for bearing sensors, $R$ is for RSSI sensors, and $F$ is for the fox.

These observations form a partial order, shown in Figure 7 (p. 666), obtained by "gluing together" several diagrams like those shown in Figures 5(a) and 6(a) along the common observations about the fox transmitter. The order relation $\leq$ is the transitive closure of the relation induced by the graph, so $R_{3}^{\prime} \leq F^{\prime} \leq F$ for instance. This 


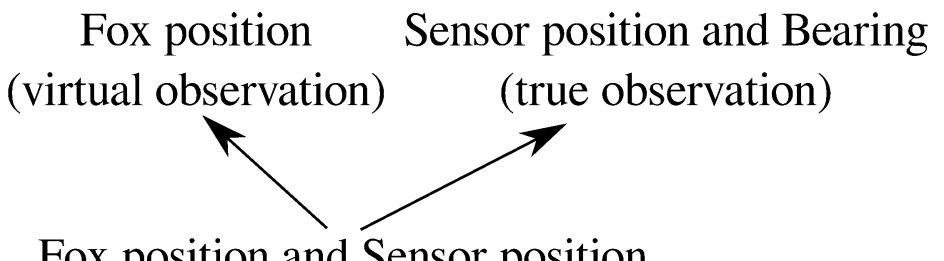

Fox position and Sensor position

(virtual observation)

(a)

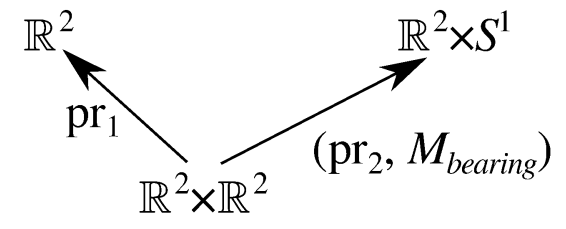

(b)

Figure 5. Measurement of a bearing to the fox: (a) partial order of the dependencies between observations, (b) the spaces of observations and the measurement functions determining them. (This is a subgraph corresponding to both $\left\{F, B_{1}^{\prime}, B_{1}\right\}$ and $\left\{F, B_{2}^{\prime}, B_{2}\right\}$ in Figure 7.)

\section{Fox position and Transmit power Sensor position and Received power

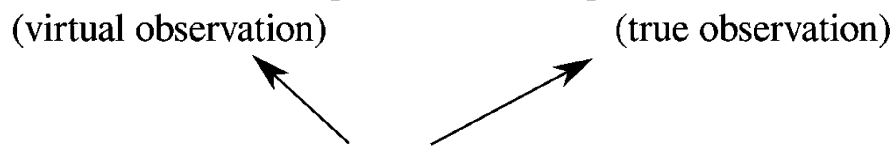

Fox position, Transmitter power, and Sensor position (virtual observation)

(a)

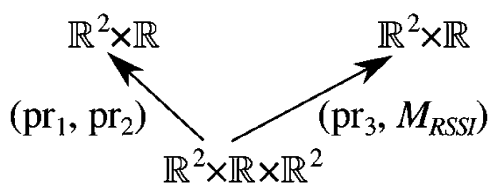

(b)

Figure 6. Measurement of an RSSI report of the fox: (a) partial order of the dependencies between observations, (b) the spaces of observations and the measurement functions determining them. (This is a subgraph corresponding to both $\left\{F^{\prime}, R_{3}^{\prime}, R_{3}\right\}$ and $\left\{F^{\prime}, R_{4}^{\prime}, R_{4}\right\}$ in Figure 7.)

implies that the observation $R_{3}^{\prime}$ functionally determines the observation $F$.

Figure 8 shows each observation's space of values and specifies each of the measurement functions, which makes all of the functional dependencies between observations explicit. Figure 8 defines a bit more than a partial order; it determines a sheaf on the partial order.
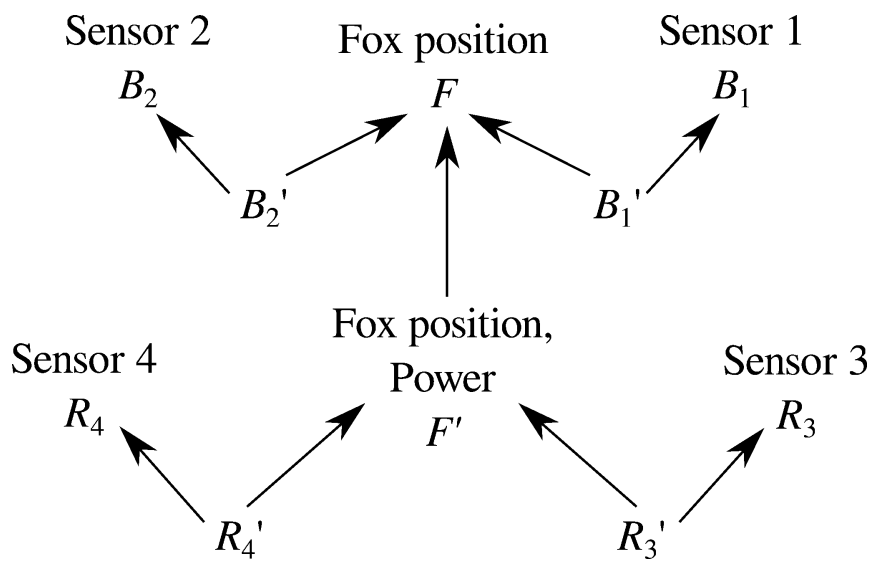

Figure 7. Partial order of all observations shown in Table 3
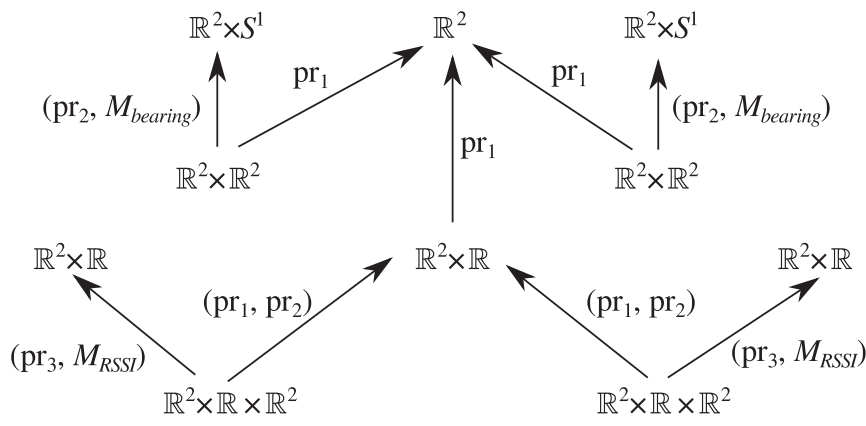

Figure 8. The sheaf diagram for all observations shown in Figure 7.

Definition 1. [1] A sheaf $S$ on a partial order $(X, \leq)$ consists of the specification of:

1. A set $S(x)$ for each $x \in X$, called the stalk on $x$, and

2. A function $S(x \leq y): S(x) \rightarrow S(y)$, called the restriction along $x \leq y$, for each $x \leq y \in X$, such that

3. Whenever $x \leq y \leq z \in X$, it follows that $S(x \leq$ $z)=S(y \leq z) \circ S(x \leq y)$.

Notice that we need not specify the restriction along $R_{3}^{\prime} \leq F$ in Figure 7, for instance, since this is already completely determined by the composition of restrictions 
Table 4. Case 1: Known fox at location $(0.5,0.5)$

\begin{tabular}{|c|l|l|}
\hline Observation & Description & Value \\
\hline \hline$F^{\prime}$ & Fox position, transmit power & $(0.5,0.5), 1.0$ \\
\hline$F$ & Fox position & $(0.5,0.5)$ \\
\hline$B_{1}^{\prime}$ & Fox position, Sensor 1 position & $(0.5,0.5),(1.0,0.0)$ \\
\hline$B_{1}$ & Sensor 1 position and bearing & $(1.0,0.0),-45$ \\
\hline$B_{2}^{\prime}$ & Fox position, Sensor 2 position & $(0.5,0.5),(1.0,1.0)$ \\
\hline$B_{2}$ & Sensor 2 position and bearing & $(1.0,1.0),-135$ \\
\hline$R_{3}^{\prime}$ & Fox position and power, Sensor 3 position & $(0.5,0.5), 1.0,(0.0,0.0)$ \\
\hline$R_{3}$ & Sensor 3 position and RSSI & $(0.0,0.0), 0.16$ \\
\hline$R_{4}^{\prime}$ & Fox position and power, Sensor 4 position & $(0.5,0.5), 1.0,(0.5,1.0)$ \\
\hline$R_{4}$ & Sensor 4 position and RSSI & $(0.5,1.0), 0.32$ \\
\hline
\end{tabular}

along $R_{3}^{\prime} \leq F^{\prime}$ and $F^{\prime} \leq F$. If we have obtained the fox position and Sensor 3 position (observation $R_{3}^{\prime}$ ), then we automatically know the fox position (observation $F$ ).

An important feature of a sheaf on a partial order is that the upward set $U_{X}$ for an element $x \in X$, given by

$$
U_{x}=\{y \in X: x \leq y\}
$$

is the set of observations $y \in U_{X}$ that are functionally determined by the observation $x$.

The collection of upward sets forms the base for a topology, called the Alexandrov topology $\operatorname{Alex}(X, \leq)$. This suggests that we ought to assign measured quantities to open sets of observations, not just individual observations.

Remark 1. The reader who is familiar with the usual definition of a sheaf on a topological space may notice that our definition of a sheaf $S$ on a partial order $(X, \leq)$ is merely a functor from the category generated by the partial order, and that the gluing axiom is apparently missing. The gluing axiom ensures that the value of $S$ on a typical open set $U$ is the space of sections for any open cover of $U$. Since we have only defined $S$ on the upward sets, not all open sets, we may use the gluing axiom to define sections and the rest of the sheaf accordingly.

Definition 2. Suppose that $U$ is an open set in $\operatorname{Alex}(X, \leq)$. The set of sections on $U$ of a sheaf $S$ on $(X, \leq)$ is

$$
\begin{array}{r}
S(U)=\left\{s \in \prod_{x \in U} S(x): s(y)=S(x \leq y)(s(x)),\right. \\
\left.\quad \text { for all } x \in U \text { and } y \in U_{x}\right\},
\end{array}
$$

namely the set of values from each stalk in $U$ that are consistent with all restrictions. A section on $X$ is called a global section.

It is immediate that $S(x)$ is in one-to-one correspondence with $S\left(U_{X}\right)$.

Case 1: All sensors, known fox, no noise. Suppose that the four sensors receive (with no noise) reports from a fox located at $(0.5,0.5)$ transmitting with power level 1.0. This corresponds to the situation of a virtual observation
$F^{\prime}$ of $((0.5,0.5), 1.0)$. Table 4 shows what all of the observations (both true and virtual) would be for this situation (positions of the sensors are from Figure 4). These observations form a global section $g$ of the sheaf $S$ shown in Figure 8. Although it is tedious to verify that $g$ really is a global section, it is enlightening to check a few restrictions. The virtual observation for the bearing Sensor 1 (observation $B_{1}^{\prime}$ ) is consistent with the fox location (observation $F$ ) because

$$
\begin{aligned}
(S & \left.\left(U_{B_{1}^{\prime}} \subset U_{F}\right)\right) g\left(B_{1}^{\prime}\right) \\
& =\left(S\left(U_{B_{1}^{\prime}} \subset U_{F}\right)\right)((0.5,0.5),(1.0,0.0)) \\
& =\operatorname{pr}_{1}((0.5,0.5),(1.0,0.0)) \\
& =(0.5,0.5)=g(F) .
\end{aligned}
$$

The observation at $B_{1}^{\prime}$ is also consistent with the bearing Sensor's true observation $B_{1}$ because

$$
\begin{aligned}
\left(S\left(U_{B_{1}^{\prime}} \subset U_{B_{1}}\right)\right) g\left(B_{1}^{\prime}\right) & \\
= & \left(S\left(U_{B_{1}^{\prime}} \subset U_{B_{1}}\right)\right)((0.5,0.5),(1.0,0.0)) \\
= & \left(\operatorname{pr}_{2}((0.5,0.5),(1.0,0.0)),\right. \\
& \left.M_{\text {bearing }}(0.5,0.5,1.0,0.0)\right) \\
= & ((1.0,0.0),-45)=g\left(B_{1}\right) .
\end{aligned}
$$

The sheaf structure imposes constraints on sections over unions of overlapping upward sets. Observations $B_{1}^{\prime}$ and $B_{2}^{\prime}$ both stipulate a position for the fox transmitter in their first factor, but $F$ also stipulates a position for the fox transmitter. The model posited by a section over $U_{B_{1}^{\prime}} \cup U_{B_{2}^{\prime}}$ argues that there is only one transmitter, so each of these positions ought to be the same.

So while $S\left(B_{1}^{\prime}\right)=S\left(U_{B_{1}^{\prime}}\right)=\mathbb{R}^{4}$ represents the fox position and Sensor 1 position, and $S\left(B_{2}^{\prime}\right)=S\left(U_{B_{2}^{\prime}}\right)=\mathbb{R}^{4}$ represents the fox position and Sensor 2 position, their intersection $S(F)=S\left(U_{F}\right)=\mathbb{R}^{2}$ represents the fox position only. A section on the union

$$
U_{B_{1}^{\prime}} \cup U_{B_{2}^{\prime}}=\left\{B_{1}^{\prime}, B_{1}, B_{2}^{\prime}, B_{2}, F\right\}
$$


asserts that the fox positions between the two sensors are held in common, so that $S\left(U_{B_{1}^{\prime}} \cup U_{B_{2}^{\prime}}\right)=\mathbb{R}^{6}$ by the inclusion-exclusion principle.

Moreover, any global section is determined by its value on each of the minimal elements of the partial order in Figure 7. For this particular sheaf, specifying the fox position, fox transmitter power, and the positions of each of the four sensors completely determines a global section. Thus the set of global sections is $\mathbb{R}^{11}=\mathbb{R}^{2} \times \mathbb{R} \times\left(\mathbb{R}^{2}\right)^{4}$.

\section{Consistency Radius: Where is the Fox?}

Sections on large open sets are rarely observed due to a variety of uncertainties about the model and the presence of stochastic noise. One cannot detect these uncertainties from a single observation; they are only apparent after comparing with other observations. This leads to the notion of an assignment, where the consistency described by restriction functions is not enforced.

Definition 3. [13]. For a sheaf $S$ on a partial order $(X, \leq)$ with the Alexandrov topology $\mathcal{T}=\operatorname{Alex}(X, \leq)$, and a collection of open sets $\mathcal{U} \subseteq \mathcal{T}$, an assignment supported on $\mathcal{U}$ is an element of $\prod_{U \in \mathcal{U}} S(U)$.

If the space of sections $S(U)$ is a pseudometric space with pseudometric $d_{U}$ for each open $U \in \mathcal{U}$, the set of assignments supported on $\mathcal{U}$ has an assignment pseudometric for two assignments $a, b$ in $\prod_{U \in \mathcal{U}} S(U)$ given by

$$
D(a, b)=\sqrt{\sum_{U \in \mathcal{U}} d_{U}(a(U), b(U))^{2}} .
$$

Suppose that $V \subseteq W$ are two open sets in $\mathcal{T}=$ $\operatorname{Alex}(X, \leq)$. This means that $\prod_{y \in V} S(y) \subseteq \prod_{x \in W} S(x)$, so that there is a natural projection $S(W) \rightarrow S(V)$ that merely discards the stalks that are in $W$ but not in $V$. In the case of upward sets $U_{y} \subseteq U_{x}$, this projection is precisely the restriction $S(x \leq y)$. Without complicating our notation, we may unambiguously refer to the natural projection of sections $S(W) \rightarrow S(V)$ as a restriction $S(V \subseteq W)$.

Definition 4. For an assignment supported on all open sets, the consistency radius

$c_{S}(a, \mathcal{T})=\sqrt{\sum_{V \in \mathcal{T}} \sum_{U \subseteq V \in \mathcal{T}} d_{U}((S(U \subseteq V)) a(V), a(U))^{2}}$

quantifies how far a given assignment is from being a global section.

Effective usage of sheaves in practice can hinge on careful weighting among the pseudometrics in Definition 4, but equal weights among the different pseudometrics in their natural units works well enough in our fox hunting examples.

Open question 1. How should one choose the weights for the assignment pseudometric and the consistency radius?
Implicit in both the assignment pseudometric and consistency radius is a choice of norm that aggregates multiple pseudometrics. Since different norms respond differently to outliers, what guides the selection of that norm?

The central relationship between global sections of $S$ and assignments is captured by the following bound, which interprets consistency radius as an obstruction to an assignment being a section.

Proposition 1. [13, Prop. 23] For an assignment a to a sheaf $S$ of pseudometric spaces on $(X, \mathcal{T})$ in which each restriction map of $S$ is Lipschitz with constant $K$, then for every global section $S$ of $S(X)$,

$$
c_{S}(a, \mathcal{T}) \leq(1+K) D(a, s) .
$$

Strictly speaking, none of the restriction maps in this article are Lipschitz since their derivatives become unbounded when the fox and receiver approach each other. It is practical, however, to assume that the fox and the receivers are always separated by some minimum distance. This minimum distance establishes the Lipschitz constant $K$.

Case 2: Bearings only, unknown fox, no noise. To explore how consistency radius can help locate a fox transmitter, consider Sensor 1 and 2 in Figure 9(a) (p. 669), both of which are bearing sensors. Sensor 1 reports a bearing of $-45^{\circ}$ (corresponding to $B_{1}$ in Figure 9(b)) and Sensor 2 reports $-135^{\circ}$ (corresponding to $B_{2}$ in Figure 9(b)). Merely by intersecting sight lines from these sensors, we can infer that the fox is located at $(0.5,0.5)$. The sheaf of observations also recovers this information and a little more besides. The two sensors correspond to five observations shown in Figure 9(b), and this collection of observations forms the sheaf shown in Figure 9(c) when associated to their measurement functions. As before, the fox position $F$ and both joint observations $B_{1}^{\prime}, B_{2}^{\prime}$ are virtual observations, because we do not know their values.

We can encode the bearing reports as an assignment $a$ shown in Figure 10(a) (p. 669), where we note that the fox location can vary

$$
a\left(U_{F}\right)=(x, y),
$$

because we do not yet know its location. The true observations from the two sensors are given by

$$
\begin{gathered}
a\left(U_{B_{1}}\right)=((1.0,0.0),-45), \\
a\left(U_{B_{2}}\right)=((1.0,1.0),-135),
\end{gathered}
$$

since we know both the sensor locations and the bearings. The two virtual observations at $B_{1}^{\prime}$ and $B_{2}^{\prime}$ are typically over constrained by these three facts because most points $(x, y)$ in the plane do not satisfy the simultaneous system

$$
\begin{gathered}
M_{\text {bearing }}(x, y, 1.0,0.0)=-45, \\
M_{\text {bearing }}(x, y, 1.0,1.0)=-135 .
\end{gathered}
$$




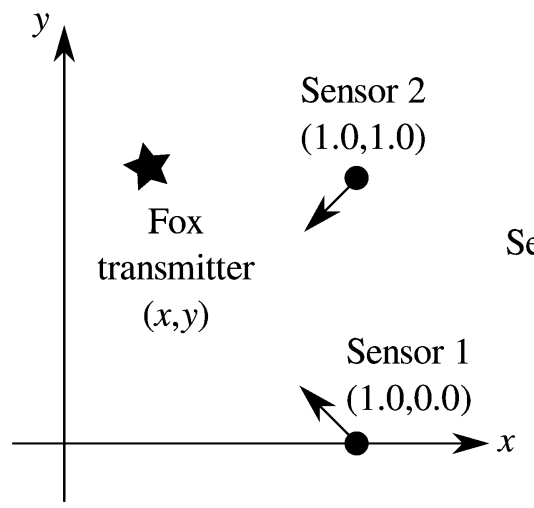

(a)

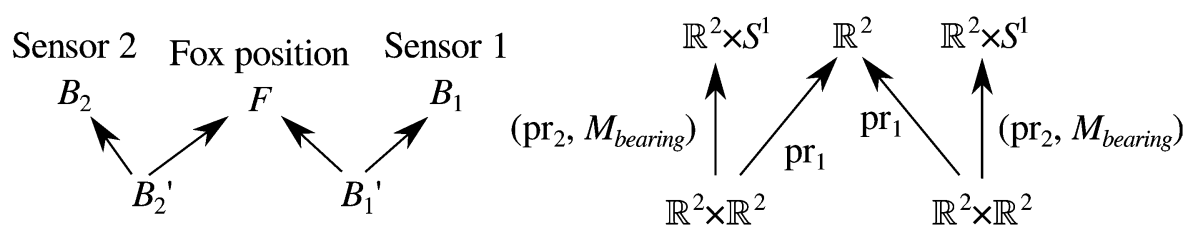

(b)

(c)

Figure 9. The setup for Case 2: (a) the spatial layout of the two bearing sensors and the fox whose location is unknown, (b) the partial order of the observations, and (c) the sheaf diagram.

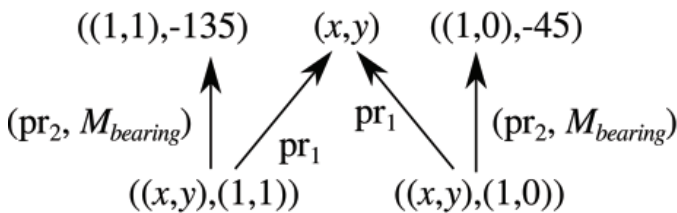

(a)

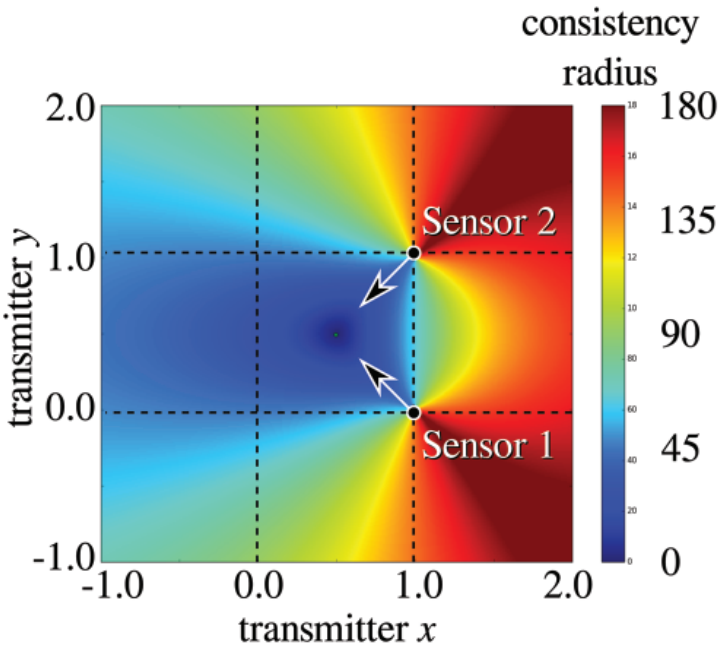

(b)

Figure 10. The results of Case 2: (a) an assignment to the sheaf in Figure 9(c) with Sensor 1 receiving a bearing of $-45^{\circ}$ and Sensor 2 receiving a bearing of $-135^{\circ}$, (b) consistency radius as a function of fox transmitter position. Notice the prominent minimum where the bearing sight lines (marked with arrows) coincide.

Therefore, the consistency radius of the assignment $a$ is typically not zero. Figure 10(b) shows the consistency radius of $a$ as a function of the fox position $(x, y)$. Two conclusions can be drawn immediately from this plot: (1) the point of intersection $(0.5,0.5)$ between the two sight lines minimizes the consistency radius (zero), (2) no other fox transmitter position will yield an assignment with consistency radius of zero. This strongly points to the conclusion that the fox transmitter is located at $(0.5,0.5)$.

Optimally extending assignments. Case 2 above indicates that minimizing the consistency radius is a useful inference procedure. Instead of encoding the signal reports from the sensors as an assignment with values at both true and virtual observations, we really only need to consider values at the true observations. For instance, if we did not specify values at $B_{1}^{\prime}, B_{2}^{\prime}$, and $F$ in Figure 9(b), we could infer them from the values at $B_{1}$ and $B_{2}$. This warrants a more general definition of consistency radius.

Definition 5. The consistency radius of an assignment a supported on $\mathcal{U}$-rather than all open sets-is the infimum of all consistency radii of assignments $b$ that restrict to $a$, namely

$$
\begin{aligned}
& c_{S}(a, \mathcal{U}) \\
& =\inf \left\{c_{S}(b, \mathcal{T}): b \in \prod_{V \in \mathcal{T}} S(V)\right. \\
& \quad \text { such that } b(U)=a(U) \text { whenever } U \in \mathcal{U}\} .
\end{aligned}
$$


We say that each such assignment $b$ extends $a$.

A nonzero consistency radius for an assignment that is supported on $\mathcal{U}$, rather than on all open sets, is still the obstruction to extending that assignment to a global section, as the next Proposition states.

Proposition 2. [14, Prop. 11] If $a$ is an assignment to a sheaf $S$ on a topological space $(X, \mathcal{T})$ supported on $\mathcal{T}$ (every open set) and $U \in \mathcal{T}$, then

$$
c_{S}(a, \mathcal{T} \cap U) \geq d_{V_{1}}\left(S\left(V_{1} \subseteq V_{2}\right) a\left(V_{2}\right), a\left(V_{1}\right)\right)
$$

for every $V_{1} \subseteq V_{2} \subseteq U$.

Case 3: All sensors, unknown fox, noise present. Consider the situation of trying to infer the location of a single unknown fox transmitter using all four sensors, in which the reports are contaminated with noise. Several simulations were run with different amounts of noise and different random draws to produce signal reports for each sensor. Each simulation produced four values, $\left(\theta_{1}, \theta_{2}, r_{3}, r_{4}\right)$ for the bearing from sensor 1 , the bearing from sensor 2, RSSI from sensor 3, and RSSI from sensor 4, respectively.

These four sensors' reports correspond to the open set $\left\{B_{1}, B_{2}, R_{3}, R_{4}\right\}$ in the partial order of the observations shown in Figure 11(a) (p. 671). The simulated reports were encoded as an assignment to the sheaf shown in Figure 11 (b) supported on the open set $\left\{B_{1}, B_{2}, R_{3}, R_{4}\right\}$. Notice that the true observations-within the support of the assignment-are shown by an element of the stalk specifying the signal report, while the figure shows the entire stalk at each virtual observation.

Each simulation therefore corresponds to an assignment, for which we would like to compute consistency radius using Definition 5. This requires finding an extension of each assignment with minimal consistency radius. This extension was found using the "SLSQP" method of the popular SciPy optimizer scipy . optimize.minimize, which can solve general optimization problems. The default options for stopping conditions were sufficient in all our simulations, but more sophisticated optimizers or careful selection of parameters is sometimes required for other sheaves [15].

Figure 12 (p. 671) shows how consistency radius depends on stochastic noise present in simulated signal reports. In Figure 12(a), no noise is present in the reports from Sensor 3 and 4 (RSSI reports $r_{3}$ and $r_{4}$ ) but is present in the reports from Sensors 1 and 2 (bearing reports $\theta_{1}$ and $\theta_{2}$ ). The consistency radius generally increases steadily as the amount of noise in the bearing reports increases. Figure 12(b) shows the same situation but the bearings are noiseless, while noise is applied to the RSSI reports. The increase in consistency radius is somewhat more abrupt, but stabilizes for larger noise values.
The fox location appears in several different places in an assignment supported on all observations, namely $B_{1}^{\prime}$, $B_{2}^{\prime}, R_{3}^{\prime}, R_{4}^{\prime}, F^{\prime}$, and $F$. Since

$$
U_{F}=\{F\}=U_{B_{1}^{\prime}} \cap U_{B_{2}^{\prime}} \cap U_{R_{3}^{\prime}} \cap U_{R_{4}^{\prime}} \cap U_{F^{\prime}},
$$

we deem that the predicted fox location is specified by the (virtual) observation at $F$.

Even though a given extension of an assignment may minimize consistency radius, it may not predict the correct location of the fox. Figure 13 (p. 672) shows the errors for the inferred location of the fox transmitter. The figure shows results from two ways to infer virtual observations when presented with a set of true observations as an assignment $a$ supported on $\mathcal{U}$ : (1) extension: find the assignment extending $a$ to all open sets that minimizes consistency radius (green triangles), as given by Definition 5 or (2) fusion: find the nearest global section $s$ to $a$ in the assignment pseudometric (red dots) [13]. In both cases, the overall transmitter location error is largely independent of noise level, with angle errors being more detrimental. The green triangles in Figure 13 correspond to the same simulations shown in Figure 12, but the vertical scales are different: consistency radius is not the same as location error.

Open question 2. Solving the extension problem generally results in substantially better estimates of the fox transmitter location than solving the fusion problem, which is why we discuss it here. While this intriguing phenomenon has been observed in other settings [15], what are the precise conditions under which extension outperforms fusion?

\section{Local Consistency Radius: Finding Multiple Foxes}

The traditional amateur radio fox hunt involves only one fox transmitter. If there are actually multiple fox transmitters and the sensors are in general position, the minimum consistency radius of an assignment constructed from the signal reports will not be zero. Sheaves allow us to segment the observations into those of different foxes. This deduction can be fully justified if we can identify open sets of sensors whose reports are consistent, even when the collection of all sensors is not. The extent to which consistency is obtained on an open set is formalized by the following definition.

Definition 6. Let $a$ be an assignment to every open set in a sheaf $S$ of pseudometric spaces on $(X, \mathcal{T})$. For an open $U \in \mathcal{T}$, the local consistency radius on $U$ is

$$
\begin{aligned}
& c_{S}(a, U) \\
& :=\sqrt{\sum_{V_{2} \subseteq U \in U, V_{1} \subseteq V_{2} \in U} d_{V_{1}}\left(\left(S\left(V_{1} \subseteq V_{2}\right)\right) a\left(V_{2}\right), a\left(V_{1}\right)\right)^{2}} .
\end{aligned}
$$

Using this, the $\epsilon$-consistent collection consists of every connected open set $U \in \mathcal{T}$, such that $(1) c_{S}(a, U)<\epsilon$ and 


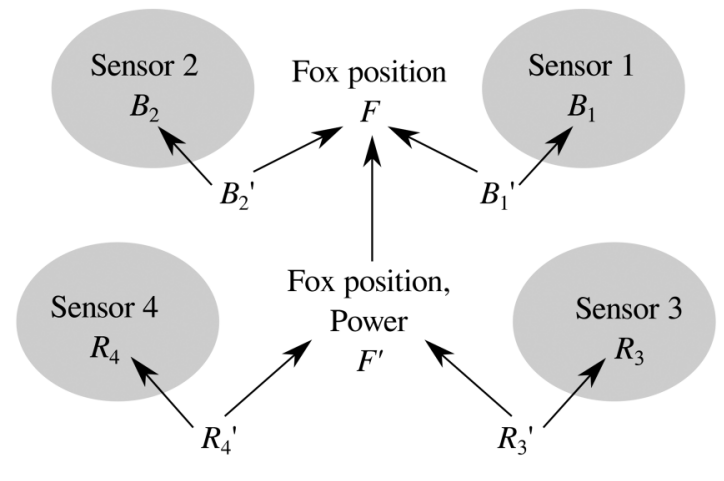

(a)
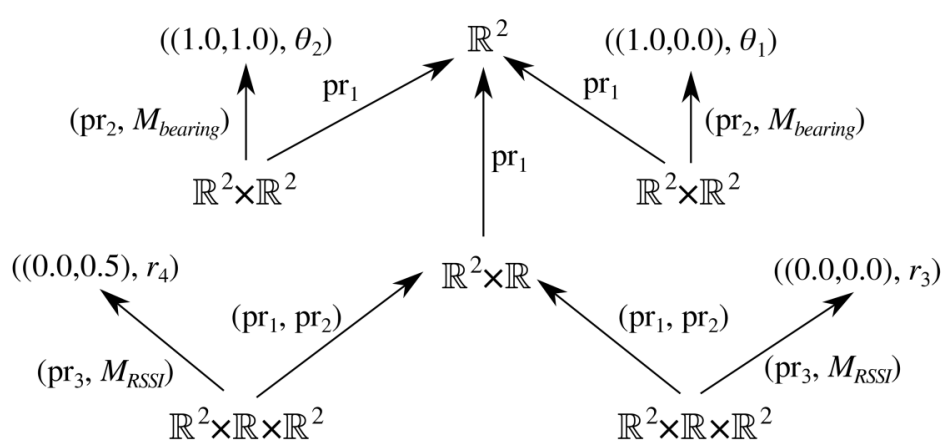

(b)

Figure 11. Case 3: Inferring fox location from reports from all sensors. (a) The partial order of observations (true observations are shaded), and (b) the resulting assignment supported on the true observations. Virtual assignments are marked with the stalk from the sheaf in Figure 8 for easy reference.

Consistency radius as a function of stochastic noise

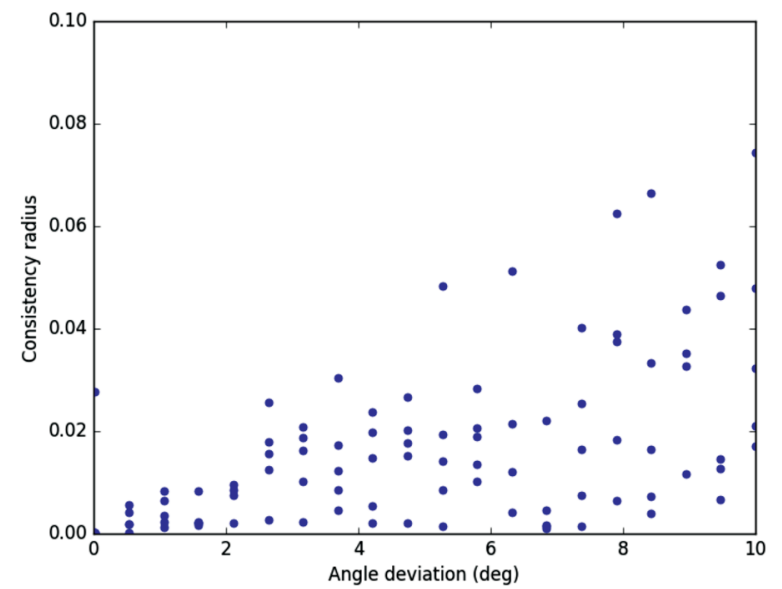

(a)

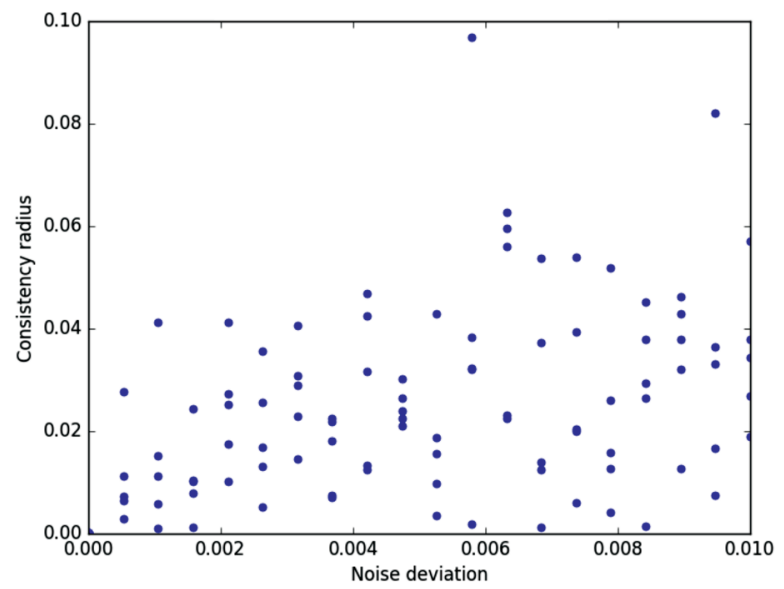

(b)

Figure 12. Consistency radius as a function of input noise level for Case 3. Each point is a separate simulation consisting of a report from each Sensor. (a) Noise is present only in Sensors 1 and 2 (bearing) according to (4). (b) Noise is present only in Sensors 3 and 4 (RSSI) according to (2).

(2) there is no other connected open set $V \in \mathcal{T}$ with $c_{S}(a, V)<\epsilon$ and $U \subset V$.

Usually, $\epsilon$-consistent collections only cover part of the space $X$. All observations $x \in X$ that are maximal in the partial order of observations will necessarily have $c_{S}\left(a, U_{X}\right)$ $=0$ for any assignment, so their upward sets are always included in any $\epsilon$-consistent collection for $\epsilon>0$. Connectedness in Definition 6 is for ease of interpretation: two observations lying in different connected components of an open set $U$ are never tested for consistency when computing the local consistency radius on $U$.

Lemma 1. Local consistency radius is a monotonic function of the open set. Specifically, if $a$ is an assignment to a sheaf $S$ of pseudometric spaces and $U \subseteq V$ are open subsets of the base space, then

$$
c_{S}(a, U) \leq c_{S}(a, V) .
$$

The Lemma follows from the fact that the sum in the expression for $c_{S}(a, V)$ is over a strictly larger set than the sum in the expression for $c_{S}(a, U)$.

A collection of open sets $\mathcal{V}$ is said to refine another collection of open sets $\mathcal{U}$ if every $V \in \mathcal{V}$ is contained in some $U \in U$, which can be thought of-not uniquely-as a function $\mathcal{V} \rightarrow \mathcal{U}$. Therefore, if $\tau<\epsilon$, the $\tau$-consistent collection for an assignment refines its $\epsilon$-consistent collection.

Definition 7. For an assignment $a$ to every open set in a sheaf $S$ of pseudometric spaces on $(X, \mathcal{T})$, the consistency filtration $\mathbf{C F}(S, a)$ is the set of all $\epsilon$-consistent collections for 


\section{Location errors after processing}

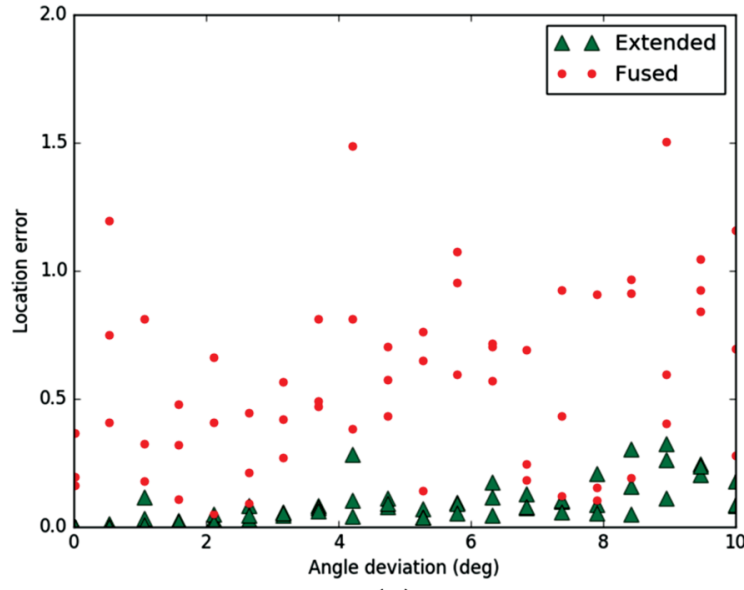

(a)

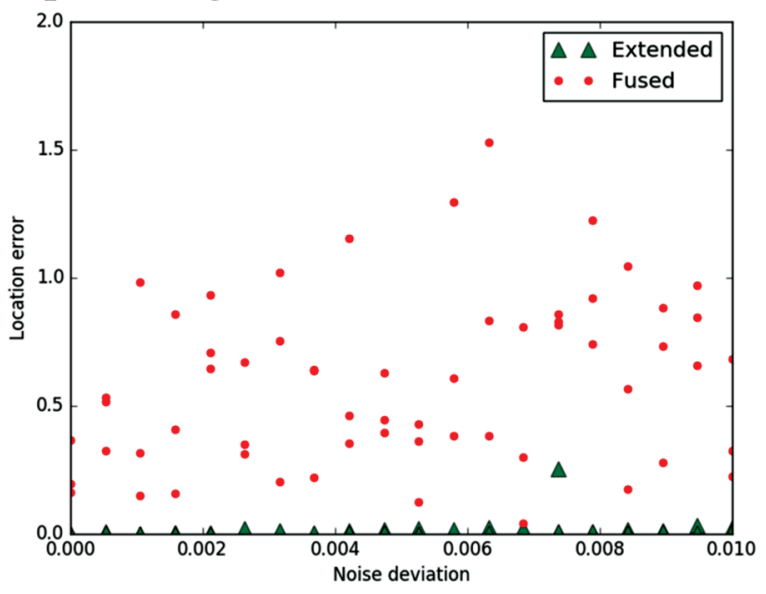

(b)

Figure 13. Fox transmitter location error after applying sheaf-based processing described in the text to Case 3 . Each point is a separate simulation consisting of a report from each Sensor. Green triangles correspond to the estimates obtained by solving the extension problem, while the red dots correspond to solving the fusion problem. (a) Noise is present only in Sensors 1 and 2 (bearing) according to (4). (b) Noise is present only in Sensors 3 and 4 (RSSI) according to (2).

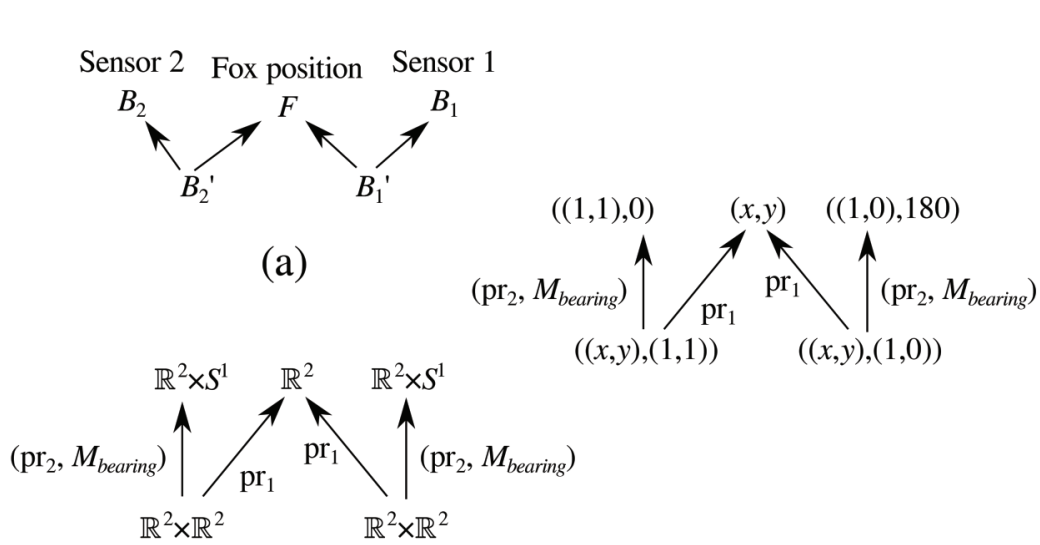

(b)

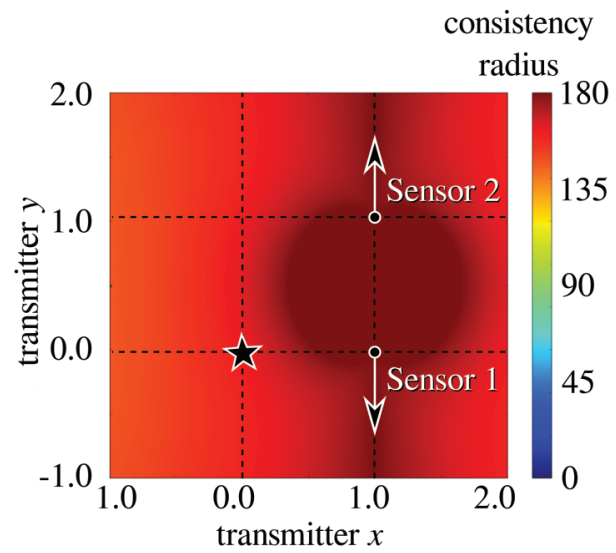

(d)

Figure 14. Case 4: Two bearing sensors receiving conflicting reports. (a) The partial order of observations, (b) the sheaf diagram, (c) the assignment representing the bearing reports shown by arrows in (d), and (d) consistency radius of the assignment as a function of fox transmitter position. The star in (d) marks the fox transmitter for the purpose of computing the consistency filtration in Figure 15 (p. 673).

all $\epsilon \in[0, \infty)$. We will use the notation $\mathbf{C F}_{(S, a)}(\epsilon)$ to refer to a particular value of $\epsilon$.

Because of Lemma 1, the consistency filtration $\mathbf{C F}(s, a)$ is actually a sheaf on the partial order $(\mathbb{R}, \leq)$, in which the restrictions $\mathbf{C F}_{(s, a)}(\tau<\epsilon)$ are refinement functions. As $\epsilon$ decreases, the open sets in $\mathbf{C F}_{(s, a)}(\epsilon)$ become more refined.

Case 4: Bearings only, two unknown foxes, no noise. Let us consider the setting in which there are two bearing sensors, shown in Figure 14. The sheaf shown in Figure 14(b) assumes that there is one fox transmitter. The setup is thus far the same as in Case 2, but instead suppose that Sensor 1 reports a bearing of $-180^{\circ}$ and Sensor 2 reports a bearing of $0^{\circ}$. This can happen if there are two foxes, but cannot happen if there is only one fox. As before, we can encode the signal reports as an assignment $a$ shown in Figure 14(c), with

$$
a\left(U_{B_{1}}\right)=((1,0),-180), a\left(U_{B_{2}}\right)=((1,1), 0) .
$$

Figure 14(d) shows the consistency radius of the assignment as a function of the fox location. The infimum consistency radius is strictly positive and is never attained, both of which indicate that there is a problem with the sheaf 


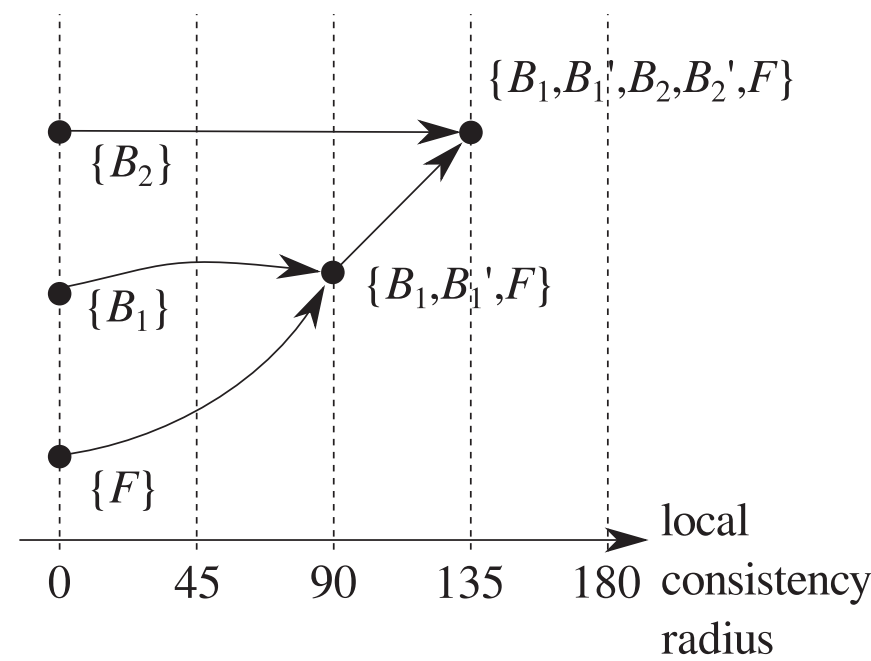

Figure 15. The consistency filtration of Case 4. Recall that Definition 6 requires only connected and maximal open sets to be shown.

model. The problem is that there is no clearly "correct" location to be inferred for the single fox transmitter.

Without computing anything, observe that the local consistency radius of $a$ on the open set $\left\{B_{1}, B_{2}, F\right\}$ is always zero, because no comparisons between these observations are required. Each of the observations $B_{1}, B_{2}$, and $F$ are perfectly self-consistent if one does not check for consistency between them! By choosing the fox location correctly at $F$, one can easily ensure that $c\left(a, U_{B_{1}^{\prime}}\right)=0$ or $c\left(a, U_{B_{2}^{\prime}}\right)=0$, but one cannot make both zero at the same time. Given this information, Lemma 1 ensures that the global consistency radius, $c\left(a, U_{B_{1}^{\prime}} \cup U_{B_{2}^{\prime}}\right)$ must be nonzero.

Definition 7 of the consistency filtration requires the assignment to be supported on every open set-since every open set is being tested for local consistency!-so let us suppose that the transmitter is located at the origin (marked with a star in Figure 14(d)). This can be encoded as the assignment shown in Figure $14(\mathrm{c})$ with $(x, y)=(0,0)$. Figure 15 shows the consistency filtration of this assignment rendered as a directed graph, in which the vertices are labeled with open sets and their local consistency radius. The edges of this graph denote subset relations between open sets.

The consistency filtration begins at $\epsilon=0$ with the three connected components $\left\{B_{1}\right\},\left\{B_{2}\right\}$, and $\{F\}$, since each of these are the largest connected open sets with consistency radius zero. $B_{1}$ and $F$ become consistent above a threshold of $90^{\circ}$ as both of these elements are subsumed into the open set $\left\{B_{1}, B_{1}^{\prime}, F\right\}$. The $\epsilon=100$-consistent collection, in which bearings are required to be closer than $100^{\circ}$, is

$$
\mathbf{C F}_{(S, a)}(100)=\left\{\left\{B_{1}^{\prime}, B_{1}, F\right\},\left\{B_{2}\right\}\right\} .
$$

Both the maximality and connectedness requirements are important: $\left\{F, B_{1}^{\prime}, B_{1}, B_{2}\right\}$ is consistent to a threshold of $100^{\circ}$ but is not connected, and while $\left\{B_{1}\right\}$ is sufficiently consistent and is connected, it is contained in a larger such set.

In Figure 15, $\left\{B_{2}\right\}$ is farther from being absorbed into a larger consistent connected open set than any of $\{F\}$, $\left\{B_{1}\right\}$, or $\left\{F, B_{1}^{\prime}, B_{1}\right\}$, which suggests that its observation is incompatible with the others. The interpretation is that Sensor 2's report is harder to reconcile with the proposed fox transmitter location than Sensor 1's report, because it is receiving a fox transmitter located somewhere else.

The consistency filtration is especially useful because it is robust to noise and other variations. If we weight the edges in the consistency filtration graph shown in Figure 15 with the difference in local consistency radius between their endpoints, we can interpret the differences between length of edges in the consistency filtration graph as the amount of noise (or other uncertainty) that we can accept and yet still detect the inconsistency between the signal reports. For instance, in order to reconcile $B_{1}$ with $B_{2}$ we must accept $135^{\circ}$ of total error: the length from $\left\{B_{2}\right\}$ to $\left\{B_{1}^{\prime}, B_{1}, B_{2}^{\prime}, B_{2}, F\right\}$. The topology of the graph is unchanged when there is less than $45^{\circ}$ of total error: the length from $\left\{B_{1}^{\prime}, B_{1}, F\right\}$ to $\left\{B_{1}^{\prime}, B_{1}, B_{2}^{\prime}, B_{2}, F\right\}$. These are guaranteed by the following theoretical result.

Theorem 1. [14, Thm. 3] For a sheaf of pseudometric spaces on a finite partial order, the consistency filtration is a continuous function of the assignment when the space of consistency filtrations is given the generalized interleaving distance [4, Def. 2.13] and the space of assignments is given the product topology.

The definition of generalized interleaving distance is rather technical, but the idea of the Theorem is straightforward. If we consider two different assignments $a$ and $b$ to a sheaf $S$ such that their consistency filtrations $\mathbf{C F}(S, a)(\epsilon)$ and $\mathbf{C} \mathbf{F}_{(S, b)}(\phi(\epsilon))$ are identical under an order preserving bijection $\phi$, then the local consistency radius of any open set cannot change more than a constant factor times the distance between the two assignments. The size of $\phi$ accounts for the difference in local consistency radii of the consistent collections, and also constrains the interleaving distance after some calculation.

Case 5: All sensors, two unknown foxes, no noise. As a final case, consider the situation in Table 5 where all four sensors are receiving noiseless signal reports, but where Sensor 3 (an RSSI sensor) receives signals from a fox located at $(0.5,0.5)$ while the other Sensors receive signals from a fox located at $(0.5,1.0)$. Both foxes use a transmit power of 1.0. If Sensor $3 \mathrm{had}$ received the same fox as the others, it would report an RSSI observation of 0.063 . Let us use the same sheaf model as in Case 1 and Case 3 


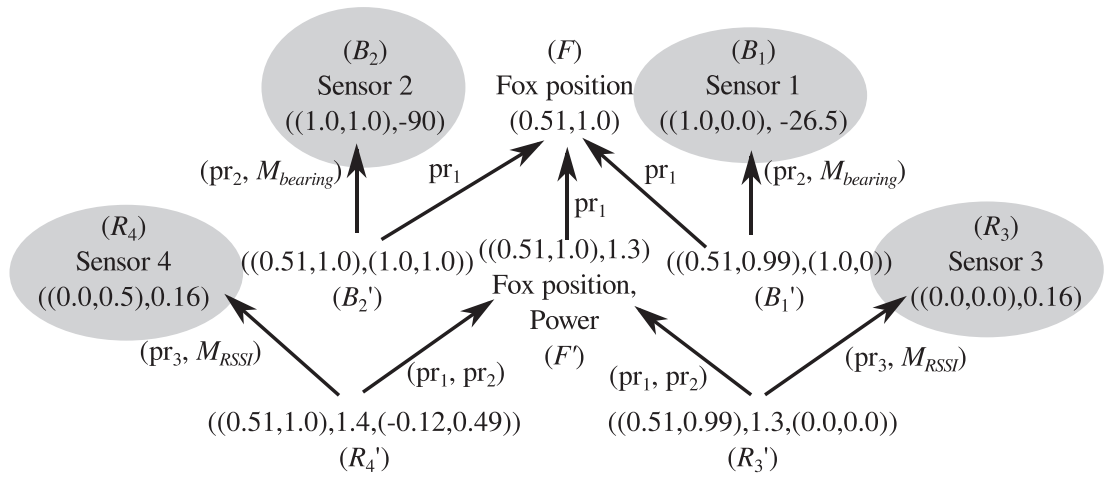

(a)

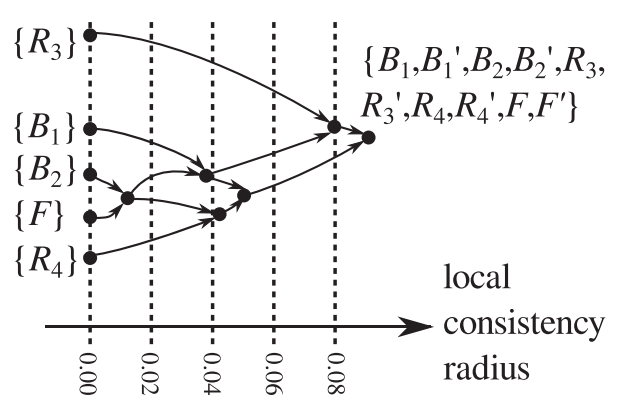

(b)

Figure 16. Case 5: all sensors and two foxes (a) The data encoded as an assignment, constructed by extending an assignment on the shaded open set to the others by way of minimizing the overall consistency radius. (b) The resulting consistency filtration of this assignment.

(the reader may refer back to Figures 8 or 11, but this is not necessary), which assumes that there is a single fox transmitter. We therefore do not expect the assignment that encodes these reports to be a global section.

The reports from Table 5 are encoded as an assignment supported on the open set $\left\{B_{1}, B_{2}, R_{3}, R_{4}\right\}$ shown shaded in Figure 16(a).

To obtain the assignment to all open sets required for the consistency filtration (Definition 7), this assignment was extended (again using "SLSQP" in Sci py . opt imize . minimize) to the one that minimizes overall consistency radius. The resulting estimates of virtual observations are shown in Figure 16(a). (Note that all open sets are not shown as this would clutter the figure considerably.)

Although there are two foxes present, the assignment predicts the location of one fox transmitter at $(0.51,1.0)$ (the observation at $F$ ), which is close to the true value of $(0.5,1.0)$ observed by Sensors 1,2 , and 4 . The consistency radius of this minimizer is 0.085 - evidently not zerowhich is a result of the conflict between Sensor 3 and the others. This is not the whole story, though. If Sensor 3 received the same fox as the others, it would report an RSSI

Table 5. Case 5 signal reports

\begin{tabular}{|c|c|c|}
\hline Sensor ID & Type & Reported observation \\
\hline \hline \multirow{2}{*}{1} & Position & $(1.0,0.0)$ \\
\cline { 2 - 3 } & Bearing & $-26.5^{\circ}$ \\
\hline \multirow{2}{*}{2} & Position & $(1.0,1.0)$ \\
\cline { 2 - 3 } & Bearing & $-90^{\circ}$ \\
\hline \multirow{2}{*}{3} & Position & $(0.0,0.0)$ \\
\cline { 2 - 3 } & RSSI & 0.16 \\
\hline \multirow{2}{*}{4} & Position & $(0.0,0.5)$ \\
\cline { 2 - 3 } & RSSI & 0.16 \\
\hline
\end{tabular}

of 0.063 instead of 0.16 . These two values have a difference of 0.097 , which is greater than the consistency radius. The discrepancy between the consistency radius and this difference is an effect of the unknown fox transmitter power. Even though both of the fox transmitters used a power of 1.0, the consistency radius is minimized with a transmit power of 1.3 (the last component of $F^{\prime}$ in Figure $16(\mathrm{a}))$, which "splits the difference" between the signal reports from Sensor 3 and Sensor 4.

Figure 16(b) shows the consistency filtration for the assignment, represented as a directed graph as was done previously in Case 4. Most of the open sets are not labeled since they can be inferred by the maximality and connectedness conditions. The portion of the diagram corresponding to Sensors 1,2 , and 4 is the open set $\left\{B_{1}, B_{1}^{\prime}, B_{2}, B_{2}^{\prime}, R_{4}\right.$, $\left.R_{4}^{\prime}, F\right\}$, which has a consistency radius of about 0.05 . It can be seen that $B_{1}$ became consistent with the predicted fox location at a higher local consistency radius than $B_{2}$ did, because of a difference in predicted fox locations at the corresponding virtual observations, $B_{1}^{\prime}$ and $B_{2}^{\prime}$. Although the predicted fox location for $R_{4}^{\prime}$ is the same as $B_{2}^{\prime}$, the difference in predicted power level at $R_{4}^{\prime}(1.4)$ from the true power level (1.0) results in a local consistency radius for $U_{R_{4}^{\prime}}$ that is higher than expected from location alone.

It is also clear that one particular open set $\left\{R_{3}\right\}$ is farthest from the rest in Figure 16(b). At a local consistency radius of about 0.08 , it becomes consistent with the bearing sensors, but not with the other RSSI sensor. Notice in particular that its predicted location of the fox transmitter $(0.51,0.99)$ is not too far from the others, but that this location does not correspond to the fox that it is receiving at $(0.5,0.5)$. The other sensors become consistent with Sensor 3 at the global consistency radius of 0.085 . The large difference in consistency between open sets containing $R_{3}^{\prime}$ and the others indicates that Sensor 3 is the cause of the comparatively high global consistency radius. 
Theorem 1 is useful because it asserts that the consistency filtration changes continuously as the assignment changes, which itself varies continuously with the signal reports. The next largest open set to contain $R_{3}$ has a consistency radius of 0.08 . All of the other true observations $B_{1}, B_{2}$, and $R_{4}$ are all contained in an open set with consistency radius 0.05 . This means that Sensor 3's report can be distorted by 0.03 before the topology of the consistency filtration graph changes. Tolerance to noise is typically expressed as a signal-to-noise ratio, with the typical fox hunting system providing a signal-to-noise ratio in excess of 10 . Sensor 3's report will be deemed inconsistent with the others provided the system maintains a signal-to-noise ratio of at least $0.16 / 0.03=5.3$, which will definitely be met by a typical fox hunting system. At such a signal-to-noise ratio, the value of $B_{2}$ will remain closest to the predicted fox location $F$ since the noise level would be not more than $0.02 / 5.3=0.004$, which is smaller than the difference in local consistency radius for any of the other open sets.

Open question 3. How should consistency filtrations be analyzed in a systematic way? Certainly they can be used for segmentation of the observations, as is done above, but how can this be done most effectively?

\section{Frontiers}

Although sheaf theory got its start as an abstract tool, purposefully beyond any application, sheaves have a number of practical applications beyond fox hunting. The interested reader may enjoy reading how sheaves are useful in the theory of computation [3, 7], network coding [2], quantum graphs [11], systems of differential equations, graphical models [12], and signal processors [10]. One can also look to more general tools from category theory to combine observations, sensors, and decisions under very general conditions $[5,6]$.

The connections between statistics, topology, and sheaves have steadfastly resisted our attempts at complete understanding. While the simulations performed in this article used realistic noise models to produce signal reports, the sheaves that processed these reports were naïvely deterministic. Theoretical guarantees are available for small perturbations of the data, which provide a measure of robustness to statistical noise, but these appear to be of limited explanatory value.

The extension of an assignment supported on some open sets to the rest of the topology has the appearance of a statistical imputation problem. Should the consistency radius play the role of a decision statistic? If so, it seems that given the stochastic models, the sheaf should aid the specification of a maximum likelihood estimate of the fox transmitter. These connections are only just starting to be explored!

\section{References}

[1] Bacławski K. Whitney numbers of geometric lattices. Adv. in Math., 16:125-138, 1975. MR0387086

[2] Ghrist R, Hiraoka Y. Applications of sheaf cohomology and exact sequences to network coding. preprint, 2011.

[3] Goguen JA. Sheaf semantics for concurrent interacting objects. Mathematical Structures in Computer Science, 2(02):159-191, 1992. MR1171509

[4] Harker S, Kramar M, Levanger R, Mischaikow K. A comparison framework for interleaved persistence modules arxiv:1801.06725, 2018.

[5] Kokar M, Tomasik J, Weyman J. Formalizing classes of information fusion systems. Information Fusion, 5:189-202, 2004.

[6] Kokar MM, Baclawski K, Gao H. Category theory-based synthesis of a higher-level fusion algorithm: An example. In Information Fusion, 2006 9th International Conference on, pages 1-8. IEEE, 2006.

[7] Lilius J. Sheaf semantics for Petri nets. Technical report, Helsinki University of Technology, Digital Systems Laboratory, 1993.

[8] Longley AG, Rice PL. Prediction of tropospheric radio transmission loss over irregular terrain. a computer method-1968. Technical report, Institute for Telecommunication Sciences Boulder, Colorado, 1968.

[9] Mardia KV, Jupp PE. Directional statistics, volume 494. John Wiley \& Sons, 2009. MR1828667

[10] Robinson M. Topological Signal Processing. Springer, January 2014. MR3157249

[11] Robinson M. Imaging geometric graphs using internal measurements. J. Differential Equations, 260:872-896, 2016. MR3411693

[12] Robinson M. Sheaf and duality methods for analyzing multi-model systems. In Novel Methods in Harmonic Analysis. Birkhäuser, 2017. MR3701273

[13] Robinson M. Sheaves are the canonical datastructure for information integration. Information Fusion, 36:208-224, 2017.

[14] Robinson M. Assignments to sheaves of pseudometric spaces arxiv: 1805.08927, 2018.

[15] Robinson M, Henrich J, Capraro C, Zulch P. Dynamic sensor fusion using local topology. In 2018 IEEE Aerospace Conference, pages 1-7. IEEE, March 2018.

ACKNOWLEDGMENT. The author would like to thank the anonymous referees for their detailed critiques and thoughtful suggestions for improving this article. He also wishes to express gratitude to the amateur radio community for continuing inspiration since his childhood. This article is based upon work supported by the Defense Advanced Research Projects Agency (DARPA) and SPAWAR Systems Center Pacific (SSC Pacific) under Contract No. N66001-15-C-4040 and the Office of Naval Research (ONR) under Contract Nos. N0001415-1-2090 and N00014-18-1-2541. Any opinions, findings and conclusions or recommendations expressed 


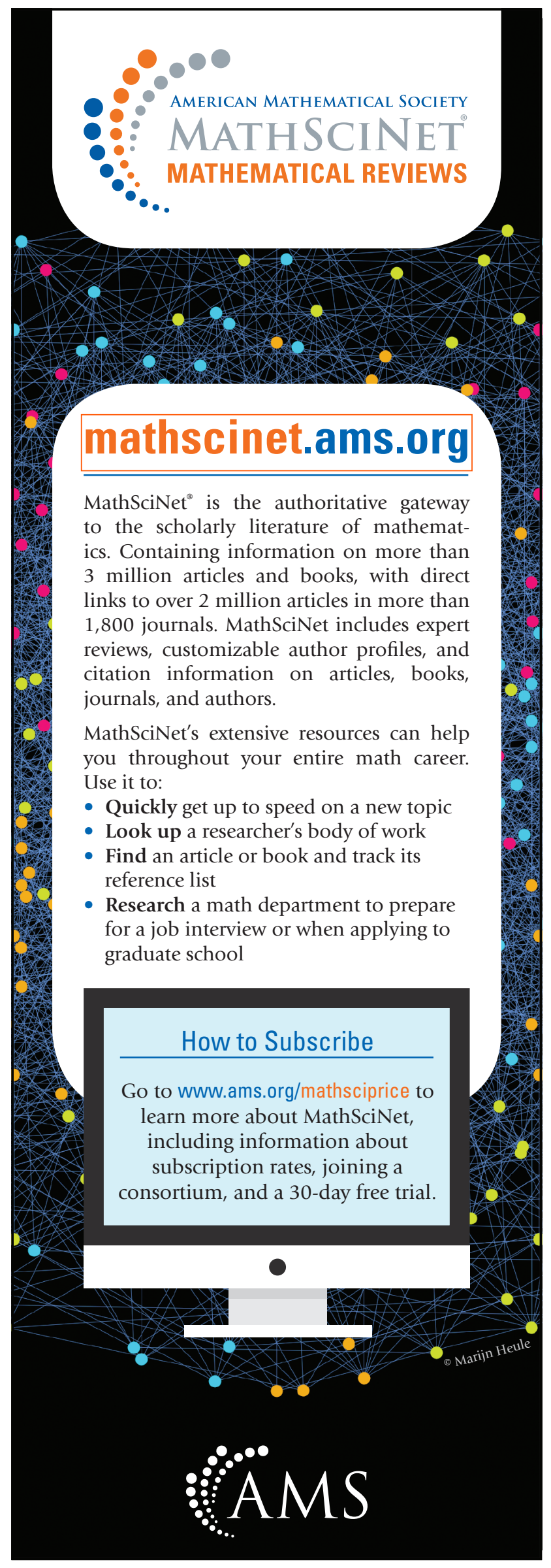

in this article are those of the author and do not necessarily reflect the views of the Defense Advanced Research Projects Agency (DARPA), SPAWAR Systems Center Pacific (SSC Pacific), or the Office of Naval Research (ONR).

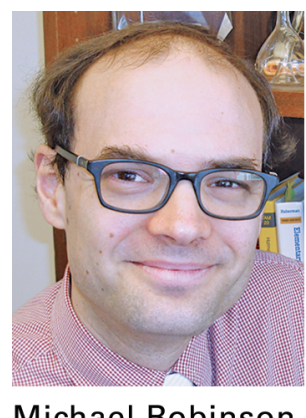

Michael Robinson

Credits

All figures are courtesy of the author. 